\title{
Riesgo de perforación en la mucosectomía esofágica con banda: estudio experimental con dos modelos de ligadores
}

\author{
J. L. Vázquez-Iglesias, P. A. Alonso-Aguirre, A. Centeno ${ }^{1}$, E. López ${ }^{1}$, T. Rodríguez y S. Blanco ${ }^{1}$ \\ Servicio de Aparato Digestivo. ${ }^{1}$ Unidad de Cirugía Experimental. Complejo Hospitalario Universitario Juan Canalejo. \\ La Coruña
}

\section{RESUMEN}

Objetivo: en los últimos años se han publicado varios trabajos que encuentran la mucosectomía con banda (MB) como un método seguro para el tratamiento de algunos tumores esofágicos, gástricos y colorrectales. Hemos realizado este estudio en animales de experimentación (cerdos) para comparar la seguridad de la MB en esófago, con dos modelos de ligadores multibanda, ya que muchos centros sólo disponen de estos modelos de ligadores comercializados para la ligadura de varices esofágicas.

Métodos: se utilizaron 8 cerdos en los que se realizaron 23 resecciones esofágicas sin inyección previa. Se hicieron 10 resecciones con el modelo Six Shooter Saeed y 13 resecciones con el modelo Speedband Superview Super 7. También se comparó la técnica realizando el corte aleatoriamente por debajo o por encima de la banda.

Resultados: se produjeron 5 perforaciones, todas con el modelo Speedband. Del total de casos en los que se utilizó este modelo se perforaron el 38,5\% frente a ninguna de las intervenciones con el modelo Six Shooter, lo que alcanzó significación estadística $(p=0,046)$. No hubo deferencias estadísticamente significativas en la frecuencia de perforación, entre realizar el corte por debajo o por encima de la banda.

Conclusiones: MB esofágica realizada con el modelo Speedband sin inyección previa, da lugar a perforación en un porcentaje elevado de casos en el animal de experimentación. Se precisan más estudios para establecer si la inyección previa incrementa la seguridad de la técnica con este modelo de ligador.

Palabras clave: Riesgo de perforación. Etudio experimental. Modelo de ligador.

\begin{abstract}
Objective: endoscopic mucosal resection with ligation (EMRL) is considered an efficient, safe method for the treatment of some esophageal, gastric and colorectal tumors. We conducted this study using a porcine model in order to compare the safety of esophageal EMRL with two multiband ligation systems, since many centers only use these ligator models in EMRL (commercialized for varix ligation).
\end{abstract}

Methods: eight pigs were used, which were submitted to 23 esophageal resections without previous injection. Ten resections were conducted using the Six Shooter Saeed model, and 13 resections used the Speedband Superview Super 7 model. The technique was also compared by making random cuts either above or below the band.

Results: five perforations occurred, all of them using the Speedband model. This represents $38.5 \%$ of total in the Speedband model group. No perforation occurred when using the Six Shooter model $(p=0.046)$. On the contrary, no significant differences were found regarding frequency of perforation when cutting above or below the band.

Conclusions: esophageal EMRL using the Speedband model without previous injection leads to perforation in a high percentage of cases in an experimental animal model. Further studies are required to find out whether a previous injection may increase the safety of this technique with this ligator model.

Vázquez-Iglesias JL, Alonso-Aguirre PA, Centeno A, López E, Rodríguez T, Blanco S. Riesgo de perforación en la mucosectomía esofágica con banda: estudio experimental con dos modelos de ligadores. Rev Esp Enferm Dig 2007; 99: 84-87.

Recibido: 08-05-06.

Aceptado:13-11-06.

Correspondencia: José Luis Vázquez-Iglesias. C/ Fernando Macías, 25-27, $7^{\circ} .15004$ La Coruña. e-mail: manitos@telefonica.net

\section{INTRODUCCIÓN}

La mucosectomía es el foco de atención de varios equipos de trabajo que la consideran un método seguro y eficaz para el tratamiento de algunos tumores del aparato digestivo. Principalmente se ha utilizado en carcinomas precoces y adenomas planos en esófago y estó- 
mago, así como en la displasia severa del esófago de Barrett (1-5). También se ha descrito como un método seguro para la extirpación de algunos tumores submucosos (6-9).

Existen diferentes modalidades de mucosectomía, pero un aspecto común a todas y paso previo a la extirpación, es la elevación y separación de la mucosa donde asienta la lesión. Para esto se usan comúnmente cuatro técnicas $(10,11)$. La más simple es la inyección de suero salino (u otra sustancia), formando un cojín por debajo de la mucosa (inyectar y cortar). En otra técnica, tras la inyección, se tracciona de la mucosa utilizando pinzas de biopsia, pero requiere endoscopios de doble canal (inyectar, traccionar y cortar). Las otras dos técnicas consisten en succionar la mucosa (y habitualmente parte de la submucosa) y se han usado con y sin la inyección previa: en algunos casos se emplea un capuchón de plástico transparente que se fija al extremo de un endoscopio convencional (mucosectomía con capuchón). La última técnica consiste en la colocación de una banda de las utilizadas en el tratamiento de varices esofágicas, y posteriormente se realiza la resección. Esta última técnica, la mucosectomía con banda (MB), se ha realizado con diferentes modelos de ligadores multibanda, tanto en el estómago como en el esófago y se ha descrito realizando el corte por encima y también por debajo de la banda (4,6-9,12-19).

Para conocer la seguridad de la técnica, específicamente en cuanto a posibilidad de perforación, hemos realizado un estudio en el animal de experimentación, con dos modelos de ligadores multibanda. Además se ha comparado la seguridad de la técnica al cortar por encima o por debajo de la banda. Estos modelos de ligadores son los más habitualmente empleados en la ligadura de varices esofágicas en España.

\section{MÉTODOS}

\section{Animales}

Se han utilizado 8 cerdos híbridos de raza Lage White con un peso entre 12 y $25 \mathrm{~kg}$. Para la realización de la MB los animales fueron anestesiados, induciendo la anestesia con ketamina y azaperona. Fueron intubados y la anestesia se mantuvo con sevoflurano $(0,5-1 \%)$ y oxígeno al $50 \%$ y la analgesia con remifentanilo en perfusión. Un mes después los animales fueron sacrificados con administración intravenosa de $\mathrm{ClK}$ tras ser anestesiados con la misma pauta descrita (o tras la propia endoscopia si en la mucosectomía se produjo perforación). Todos los procedimientos se llevaron a cabo en el quirófano de la Unidad de Cirugía Experimental del Hospital Juan Canalejo ( $\mathrm{N}^{\mathrm{o}}$ de Registro Oficial como Centro Usuario de Animales de Experimentación 15002 AE) y se han realizado siguiendo la normativa nacional y europea en el ámbito de protección y uso de animales de experimentación.

\section{Material}

Se han utilizado endoscopios Olympus CV-145 (Olympus Optical Co. Ltd. Tokio, Japan) y Fujinon EVE S200 (Fuji Photo Optical CO., Ltd. Saitama, Japan).

Se ha utilizado un electrobisturí Valleylab Force 2 (Valleylab. Boulder, CO. USA).

Se usaron dos modelos de ligador multibanda: Speedband Superview Super 7, que incorpora 7 bandas (Boston Scientific. Microvasive. Natick, MA. EE.UU.), y Six Shooter Saeed cuyo cilindro tiene montadas 6 bandas (Wilson Cook Medical. Limerick, Ireland). El modelo Speedband tiene una forma cónica con un diámetro interno proximal de 10,42 mm, diámetro interno distal de 9,42 mm y una longitud de $17,4 \mathrm{~mm}$. El modelo Six Shooter tiene una forma casi cilíndrica con un diámetro interno de 11 $\mathrm{mm}$, un diámetro distal de 9,42 mm y una longitud de 14 $\mathrm{mm}$. En la figura 1 se ven los dos dispositivos una vez utilizadas las bandas.

\section{Procedimiento de MB}

Una vez introducido el endoscopio se eligieron, al azar, los lugares para la colocación de las bandas a lo largo del esófago. Tras realizar la succión, aplicando presión negativa, se colocaron bandas de manera estándar, sin inyección previa de suero salino. La resección con asa de polipectomía se realizó aplicando corriente de mezcla por encima o debajo de las bandas de manera aleatoria. Tras la resección se valoró endoscópicamente la presencia de perforación.

\section{Método estadístico}

Las bandas utilizadas en cada animal fueron de un solo modelo. Para designar a cada animal el sistema multibanda, se hizo de manera consecutiva, comenzando al azar por el sistema Six Shooter.

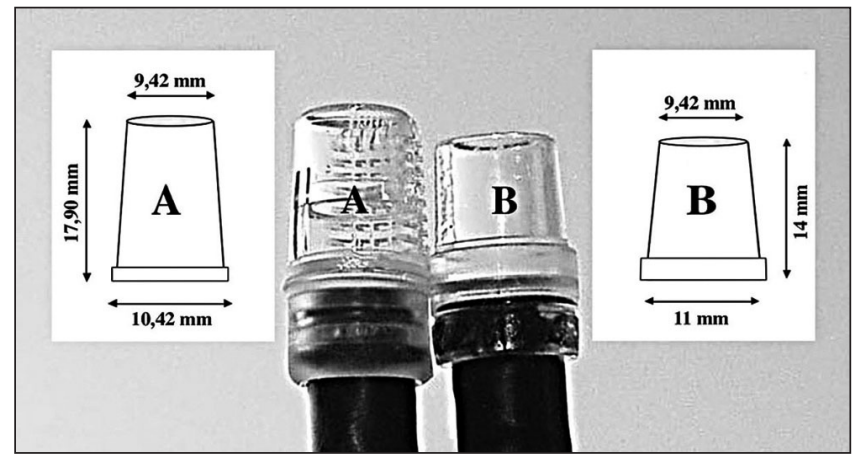

Fig. 1. Comparación de los dos modelos de ligadores, una vez que se retiraron las bandas. Obsérvese la diferencia en la longitud de los capuchones. A: Speedband Superview Super 7; B: Six Shooter Saeed. 


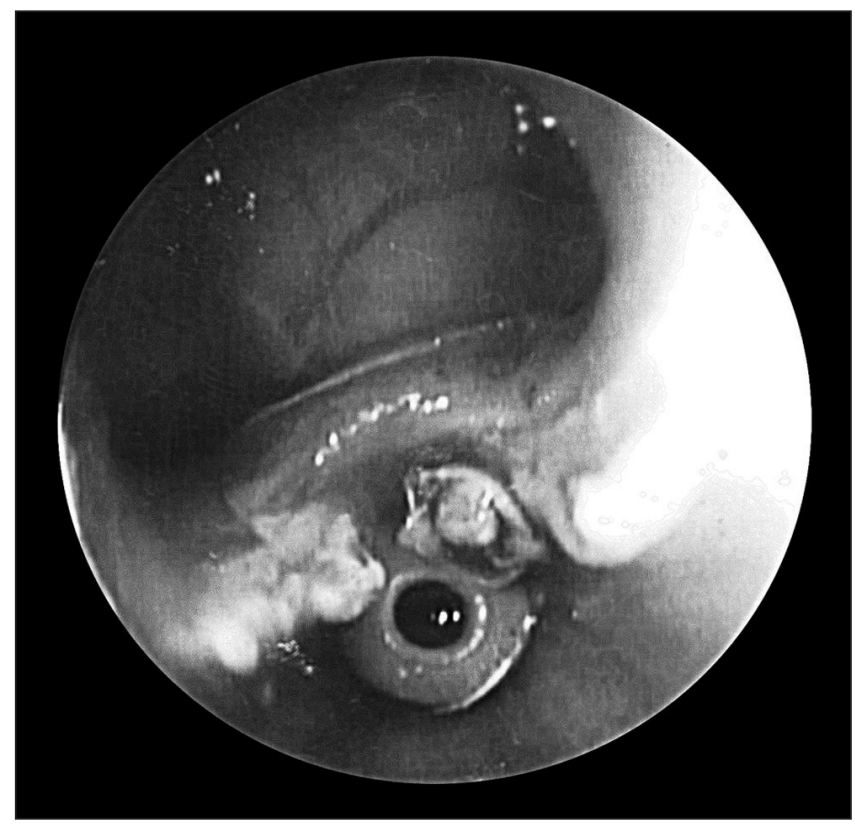

Fig. 2. Imagen endoscópica obtenida inmediatamente después de una perforación esofágica debida a mucosectomía. Se puede observar el mediastino a través de la perforación.

Se realizó un análisis descriptivo de la incidencia de perforación, según las condiciones del estudio. Los resultados se muestran como porcentajes e intervalos de confianza. Para comparar la incidencia de perforación según diferentes variables se empleó el test exacto de Fisher. Se consideraron significativos valores de $p<0,05$. Los test se hicieron con un planteamiento bilateral. El análisis estadístico se realizó con el programa SPSS 12.0 para Windows.

\section{RESULTADOS}

En la tabla I se recogen los resultados. En total se realizaron 23 intervenciones (13 con bandas modelo Speedband, 10 bandas modelo Six Shooter), de las cuales se perforaron 5 (21,7\%; 95\% IC: 8,4-41,8\%). En todos los casos en que se produjo perforación las bandas fueron del modelo Speedband. Del total de casos en los que se utilizaron bandas de ese modelo se produjo perforación en un $38,5 \%$ (5/13) (95\% IC: 15,7-65,9\%), frente a ninguna de las intervenciones en las que se utilizaron bandas del modelo Six Shooter (95\% IC: 0-25,9\%), siendo esta diferencia estadísticamente significativa $(\mathrm{p}=0,046)$. En la figura 2 se aprecia la visión endoscópica inmediata de una perforación.

Se observó menor porcentaje de perforaciones cuando el corte se realizó por encima de la banda (16,7\%; $95 \%$ IC: 2 ,9$45 \%$ versus $27,3 \%$; $95 \%$ IC 7,4-57,8\%), aunque esta diferencia no alcanzó significación estadística $(\mathrm{p}=0,64)$.
Tabla I. Resultados de las mucosectomías en función de los modelos de ligadores

\begin{tabular}{lccccc}
\hline Modelo & Cerdo $n^{\circ}$ & Resecciones & \multicolumn{2}{c}{ Corte } & Perforaciones \\
& & \multicolumn{5}{c}{ Encima } & Debajo \\
\hline Speedband & $2,4,6,8$ & 13 & 6 & 7 & 5 \\
Six Shooter & $1,3,5,7$ & 10 & 6 & 4 & 0 \\
\hline
\end{tabular}

\section{DISCUSIÓN}

Las diferentes técnicas de mucosectomía se han ido empleando cada vez con mayor frecuencia para el tratamiento de carcinomas precoces de esófago y displasia severa asociada a esófago de Barrett, hasta el punto de ser considerada la terapéutica de elección dentro de los tratamientos endoscópicos (5). A su difusión ha contribuido una baja tasa de complicaciones. La MB tiene la ventaja de que los endoscopistas están familiarizados con el dispositivo, al ser el utilizado en la ligadura de varices esofágicas.

En nuestra Unidad de Endoscopia realizamos la técnica desde hace años y comunicamos su utilidad en la resección de carcinoides rectales (20). Alguno de nuestros pacientes ha presentado dolor durante el procedimiento, inmediatamente tras la colocación de la banda y al aplicar la corriente con el asa de polipectomía. El dolor lo atribuimos a que había quedado ligada gran parte de la pared rectal. De hecho, ha sido descrita la perforación después de resección de un carcinoide rectal mediante MB (9). Esto nos llevó a plantearnos la seguridad de la técnica en el esófago, que tiene una pared más fina, con alguno de los sistemas de ligadura que se comercializan para el tratamiento de varices esofágicas y de los que disponemos en nuestra Unidad de Endoscopia.

En nuestro trabajo llama la atención el elevado porcentaje de perforaciones que contrasta con las comunicadas por grupos con gran experiencia $(4,12-15,17,18)$. La primera razón que podría explicar en parte esta frecuencia de perforación es que la pared del esófago en el animal de experimentación es de menor grosor, puesto que no tiene carcinoma. Sin embargo el grosor del esófago con displasia (asociada a esófago de Barrett ) no debe ser mayor que el esófago "sano" y hay amplia experiencia sin perforación en MB para tratamiento de la displasia de alto grado $(4,15,17)$.

Otra de las razones, que era una de las hipótesis en el planteamiento del estudio, es la posible diferencia en realizar la resección cortando por encima o debajo de la banda. Encontramos una perforación más en caso de realizar el corte por debajo, aunque sin ninguna significación estadística, si bien puede tener influencia el pequeño tamaño muestral. No obstante la técnica de MB se ha descrito indistintamente cortando por encima o debajo de la banda, sin ninguna influencia en la aparición de complicaciones $(7,10,15,16,18,19)$. 
Otra de las razones que puede explicar las perforaciones es que en nuestro protocolo no se realizó inyección previa a la resección. En otro estudio experimental en el que se siguió por toracotomía la colocación de bandas con o sin inyección, se pudo comprobar que la pared completa del esófago era succionada en caso de realizarlo sin inyección. En este estudio se produjeron 2 de 3 perforaciones en el grupo en el que no se inyectó (21). No obstante varios trabajos con numerosos pacientes realizan MB en esófago, sin inyección y mencionan la perforación como una complicación excepcional $(13,15)$.

Por otra parte la falta de inyección no explica la diferencia de perforación según el modelo de ligador utilizado. La única razón que se nos ocurre es la diferencia en el tamaño de los cilindros de los dos modelos, más concretamente en la longitud que es un $28 \%$ mayor en el modelo Speedband. Este es el modelo con el que se han producido todas las perforaciones. Este modelo lo utilizamos habitualmente en nuestra Unidad de Endoscopia para la ligadura de varices esofágicas y no hemos sufrido ninguna perforación, por lo que estamos convencidos de la seguridad del ligador para esa indicación. No obstante, en el caso de las varices esofágicas, estas se introducen en el cilindro cuando se aplica aspiración, haciendo de "cojín", e impidiendo la introducción de todo el grosor de la pared esofágica.

Ell y cols. tienen gran experiencia con otro modelo de ligador multibanda reutilizable desarrollado por su grupo, el Euroligator (22). Con este sistema que puede incorporar un total de 5 bandas han obtenido muy buenos resultados, con muy bajo porcentaje de complicaciones y sin perforación $(4,13,14,17)$. El mismo grupo ha descrito que también utiliza el modelo Speedband, pero en una versión más antigua, cuyo cilindro sólo albergaba 3 bandas y cuya longitud era mucho menor a la del modelo Speedband Super $7(4,14)$.

A la vista de los resultados de nuestro trabajo, tenemos que alertar acerca de la frecuencia de perforación esofágica en el animal de experimentación, en la MB con el modelo Speedband de 7 bandas. Se precisan más estudios para aclarar si la inyección previa puede disminuir el riesgo de esta complicación.

\section{BIBLIOGRAFÍA}

1. Makuuchi H. Endoscopic mucosal resection for mucosal cancer in the esophagus. Gastrintest Endosc Clin N Am 2001; 11: 445-58.

2. Kojima T, Parra-Blanco A, Takahashi H, Fujita R. Outcome of endoscopic mucosal resection for early gastric cancer: review of the Japanese literature. Gastrointest Endosc 1998; 48: 550-5.
3. Kudo S. Endoscopic mucosal resection of flat and depressed types of early colorectal cancer. Endoscopy 1993; 25: 455-61.

4. Ell C, May A, Gossner L, Pech O, Gunter E, Mayer G, et al. Endoscopic mucosal resection of early cancer and high-grade dysplasia in Barrett's esophagus. Gastroenterology 2000; 118 (4): 670-7.

5. May A, Gossner L, Pech O, Fritz A, Gunter E, Mayer G, et al. Local endoscopic therapy for intraepithelial high-grade neoplasia and early adenocarcinoma in Barrett's oesophagus: acute-phase and intermediate results of a new treatment approach. Eur J Gastroenterol Hepatol 2002; 14 (10): 1085-91.

6. Shikuwa S, Matsunaga K, Osabe M, Ofukuji M, Omagari K, Mizuta $\mathrm{Y}$, et al. Esophageal granular cell tumor treated by endoscopic mucosal resection using a ligating device. Gastrointest Endosc 1998; 47 (6): 529-32.

7. Ono A, Fujii T, Saito Y, Matsuda T, Lee DT, Gotoda T, et al. Endoscopic submucosal resection of rectal carcinoid tumors with a ligation device. Gastrointest Endosc 2003; 57 (4): 583-7.

8. Wehrmann T, Martchenko K, Nakamura M, Riphaus A, Stergiou N. Endoscopic resection of submucosal esophageal tumors: a prospective case series. Endoscopy 2004; 36 (9): 802-7.

9. Deist T, Kalisch C. Endoscopic resection of early rectal carcinoid tumor. Endoscopy 2004; 36 (10): 924.

10. Soetikno RM, Gotoda T, Nakanishi Y, Soehendra N. Endoscopic mucosal resection. Gastrointest Endosc 2003; 57 (4): 567-79.

11. Inoue H, Tani M, Nagai K, Kawano T, Takeshita K, Endo M, et al. Treatment of esophageal and gastric tumors. Endoscopy 1999; 31: 47-55.

12. Chaves DM, Sakai P, Mester M, Spinosa SR, Tomishige T, Ishioka S. A new endoscopic technique for the resection of flat polypoid lesions. Gastrointest Endosc 1994; 40: 224-6.

13. May A, Gossner L, Behrens A, Kohnen R, Vieth M, Stolte M, et al. A prospective randomized trial of two different endoscopic resection techniques for early stage cancer of the esophagus. Gastrointest Endosc 2003; 58 (2): 167-75.

14. Pech O, Gossner L, May A, Vieth M, Stolte M, Ell C. Endoscopic resection of superficial esophageal squamous-cell carcinomas: Western experience. Am J Gastroenterol 2004; 99 (7): 1226-32.

15. Fleischer DE, Wang GQ, Dawsey S, Tio TL, Newsome J, Kidwell J, et al. Tissue band ligation followed by snare resection (band and snare): A new technique for tissue acquisition in the esophagus. Gastrointest Endosc 1996; 44 (1): 68-72.

16. Suzuki Y, Hiraishi H, Kanke K, Watanabe H, Ueno N, Ishida M, et al. Treatment of gastric tumors by endoscopic mucosal resection with a ligating device. Gastrointest Endosc 1999; 49 (2): 192-9.

17. May A, Gossner L, Pech O, Muller H, Vieth M, Stolte M, et al. Intraepithelial high-grade neoplasia and early adenocarcinoma in shortsegment Barrett's esophagus (SSBE): Curative treatment using local endoscopic treatment techniques. Endoscopy 2002; 34 (8): 604-10.

18. Naritaka Y, Ogawa K, Shimakawa T, Wagatsuma Y, Katsube T, Kajiwara T, et al. Study on endoscopic esophageal mucosal resection with ligating device. I--Clinical study. Hepatogastroenterology 2001; 48 (40): $1015-7$.

19. Nwakakwa V, Fleischer D. Endoscopic mucosal resection of the esophagus: band ligation technique. Gastrointest Endosc Clin N Am. 2001; 11 (3): 479-88.

20. Vazquez Iglesias JL, Souto Ruzo J. A new technique for endoscopic resection of rectal carcinoid tumors. Digestion 1998; 59 (Supl. 3): 542.

21. Naritaka Y, Ogawa K, Shimakawa T, Wagatsuma Y, Katsube T, Kajiwara T, et al. Study on endoscopic esophageal mucosal resection with ligating device. II-Experimental study. Hepatogastroenterology 2001; 48 (40): 1018-21.

22. Ell C, May A, Wurster H. The first reusable multiple-band ligator for endoscopic hemostasis of variceal bleeding nonvariceal bleeding and mucosal resection. Endoscopy 1999; 31 (9): 738-40. 